Estudios Constitucionales, Año 16, No 2, 2018, pp. 89-116

ISSN 07180195

Centro de Estudios Constitucionales de Chile Universidad de Talca

"Participación de comunidades y el camino hacia un Derecho Humano al Patrimonio Cultural"

Jhonny Antonio Pabón Cadavid

\title{
PARTICIPACIÓN DE COMUNIDADES Y EL CAMINO HACIA UN DERECHO HUMANO AL PATRIMONIO CULTURAL*
}

\author{
COMMUNITIES' PARTICIPATION AND THE ROAD THROUGH A \\ Human Right to Cultural Heritage
}

\author{
Jhonny Antonio Pabón Cadavid* \\ Universidad Externado de Colombia, Victoria University of Wellington \\ pabonjhon@myvuw.ac.nz
}

RESUMEN: Las políticas multiculturales, la promoción de la diversidad cultural y el reconocimiento de múltiples identidades culturales, étnicas y politicas dentro de los Estados han propiciado que el derecho del patrimonio sea discutido dentro del contexto de los derechos humanos. La participación de comunidades (especialmente de comunidades indigenas) en los procesos de patrimonialización de sus recursos culturales ha abierto el camino hacia el reconocimiento del derecho al patrimonio cultural como un derecho humano. La Convención para la Salvaguardia del Patrimonio Cultural Inmaterial, el Convenio de Faro, el informe de la experta independiente en el campo de los derechos culturales del Alto Comisionado de las Naciones Unidas para los Derechos Humanos (ACNUDH) son los más recientes desarrollos a nivel internacional en el reconocimiento de un derecho de acceso y disfrute al patrimonio cultural. Las convenciones de la Unesco y otros instrumentos jurídicos del derecho del patrimonio elaborados en el siglo XX requieren ser actualizados en su interpretación y operación para incluir la participación efectiva de las comunidades en cuanto a la repatriación y a los procesos de nominación, designación y gestión del patrimonio mundial.

ABSTRACT: Cultural heritage law has been disengaged from cultural rights recognized in international human rights instruments. Multicultural policies, the promotion of cultural diversity and the recognition of multiple cultural, ethnic and political identities have led to the right of heritage being discussed within the context of human rights. The participation of communities (especially of indigenous communities) in heritage management has opened the way towards the recognition of the right to cultural heritage as a human right. The Convention for the Safeguarding of the Intangible Cultural Heritage, the Faro Convention, the report of the independent expert in the field of cultural

\footnotetext{
* Trabajo recibido el 8 de marzo de 2017 y aprobado el 1 de agosto de 2018.

** Ph.D. Victoria University of Wellington, Nueva Zelanda. Magister Science en Knowledge Management, Nanyang Technological University, Singapur. Magíster en Historia, Pontificia Universidad Javeriana, Colombia. Abogado, Universidad Externado de Colombia. Miembro correspondiente de la Academia Colombiana de Historia. Investigador asociado en Le Centre d'Études et de Recherche en Droit de l'Immatériel, Université Paris Sud, Francia.

El autor agradece a Felipe Michelini, Roberto Gargarella, Joss Opie y Julián Santiago Grueso por sus comentarios, críticas y sugerencias a borradores del presente artículo.
} 
rights of the United Nations High Commissioner for Human Rights (OHCHR) are the most recent developments at the international level in the recognition of a right of access and enjoyment of cultural heritage. The Unesco conventions and other legal instruments developed in the $20^{\text {th }}$ century need to be updated in their interpretation and operation to include the effective participation of communities in areas such as repatriation, nomination, designation and heritage management.

PALABRAS CLAVE: Patrimonio cultural, derechos culturales, Derechos humanos, politicas culturales. KEY WORDS: Cultural heritage, cultural rights, human rights, cultural policies.

\section{INTRODUCCIÓN}

El patrimonio está relacionado con la idea de legado; comprende tanto aquello que es recibido como lo que es transferido a las generaciones inmediatas y futuras. La primera definición de patrimonio en el Diccionario de la lengua española se refiere a lo que alguien ha heredado de sus ascendientes ${ }^{1}$. De forma similar, el diccionario de Oxford en inglés define heritage, en su primera acepción, como lo que ha sido o puede ser heredado ${ }^{2}$. Moko Mead, antropólogo maorí, resaltó que el patrimonio cultural son aquellas posesiones culturales que se transmiten activamente de generación en generación y que son valoradas por los donantes y por los receptores ${ }^{3}$. Mead enfatiza que el patrimonio se concentra en la parte de una cultura considerada tan importante que el acto de transmisión a la próxima generación se vuelve explícito y es de alta prioridad para la sociedad ${ }^{4}$. Es decir que dentro del concepto de patrimonio cultural prevalece la protección y gestión de ciertos recursos culturales, lo que conlleva a la selección y al desarrollo de medidas para lograr una transmisión intergeneracional.

El patrimonio cultural es un término anfibológico que cambia, por ejemplo, según la disciplina académica que utiliza el concepto ${ }^{5}$. Así mismo, la noción jurídica de patrimonio cultural tiene sus raíces en la tradicional institución de patrimonio del derecho civil que se relaciona con el derecho de propiedad ${ }^{6}$. De manera general, la visión civilista del patrimonio consiste en una universalidad

\footnotetext{
1 Diccionario de la lengua española (2017).

2 Oxford English Dictionary (2017).

3 Mead (1993), p. 225.

4 Mead (1993), p. 225.

5 Fairclough (2009), p. 29.

6 Cornu (2008), p. 284.
} 
jurídica de derechos y obligaciones de carácter pecuniario 7 . Siguiendo esta influencia, el patrimonio cultural ha sido definido en la ley, por ejemplo en Francia, como toda propiedad real o personal, propiedad pública o privada, de interés histórico, artístico, arqueológico, estético, científico o técnico ${ }^{8}$. Sin embargo, el término patrimonio cultural se ha utilizado en la literatura jurídica desde una perspectiva más amplia9. Prott y O'Keefe definen el alcance del patrimonio cultural como "manifestaciones de la vida humana que representan una visión particular de la vida y son testigos de la historia y validez de esa visión"10. James Nafziger, académico norteamericano, define el patrimonio cultural como "parte de una cultura nacional, tribal o de otra sociedad que es tan fundamental para la identidad y el carácter de la sociedad que se considera inalienable de ella"11. Estas definiciones amplias abarcan aspectos culturales tangibles e intangibles, incluyendo el ambiente construido y los conocimientos tradicionales.

El patrimonio cultural se crea y define dentro de medioambientes, contextos y prácticas culturales, sociales, políticas y económicas específicas. Las múltiples definiciones y el alcance del significado del patrimonio cultural están delineados por las necesidades de cada época y de la sociedad en momentos históricos concretos $^{12}$.

Hoy en día el patrimonio cultural comprende objetos y acciones, productos y procesos, y su enfoque se centra en significados y prácticas más que en artefactos ${ }^{13}$. Estos significados son creados por la interacción, el consumo y la recepción de información y experiencias ${ }^{14}$. Por tanto, el patrimonio va más allá de los artefactos culturales; el patrimonio cultural se relaciona con la creación y configuración de la identidad y los recuerdos colectivos e individuales a través de la interacción entre los restos tangibles e intangibles del pasado común y las experiencias vivas. Esta comprensión desdibuja los límites materiales e inmate-

\footnotetext{
7 Figueroa (1997), p. 49.

8 Code du Patrimoine, art. L1.

9 En 1989, Lyndel V. Prott, una destacada académica australiana en el campo del derecho del patrimonio cultural subrayó la confusión de las definiciones en la literatura jurídica, ver ProtT (1989), p. 224.

10 Prott y O’Keefe (1992), p. 307.

11 Nafziger (2008), p. 168.

12 Graham (2002), p. 1004.

13 Fairclough (2009), p. 29.

14 Graham (2002), p. 1004.
} 
riales del patrimonio, la idea de lo tradicional y nuevo, y traslada el enfoque del patrimonio como evocación del pasado hacia una perspectiva donde la consideración del presente y el futuro son esenciales ${ }^{15}$.

La intervención legal en el patrimonio cultural es parte de la gobernanza de los recursos culturales claves para la identidad de las comunidades e individuos. El debut del concepto contemporáneo de patrimonio cultural se da durante la Revolución francesa para agrupar objetos y monumentos heredados de épocas pasadas que el Estado optó preservar en razón de un interés, especialmente por su valor histórico o artístico. De manera que la idea de un patrimonio nacional surge como un instrumento del Estado francés para eliminar diferencias culturales y construir un imaginario de una comunidad homogénea. Las políticas patrimoniales contemporáneas tienen su origen entonces en el afianzamiento de la asimilación cultural dentro de la construcción monocultural del Estadonación.

Las políticas multiculturales que surgieron durante la segunda mitad del siglo XX han alterado los discursos dominantes del Estado-nación como unidades culturales homogéneas y estáticas. El reconocimiento político de la existencia de Estados biculturales como Nueva Zelanda y constituciones multiculturales o plurinacionales, como en el caso de Bolivia y Ecuador, generan nuevas dinámicas en la gestión y políticas del patrimonio cultural. En ese contexto, la participación de diversas comunidades es cada vez mayor en las políticas de la identidad a nivel local, nacional e internacional.

El presente artículo examina la hipótesis de que el reconocimiento y participación de diversas comunidades culturales, étnicas y políticas, especialmente comunidades indígenas, están reconfigurando el derecho del patrimonio cultural para insertarlo dentro del contexto de los derechos humanos. Este movimiento obedece a una apertura para que diferentes grupos participen en las políticas de la identidad del Estado-nación. Para ello, el presente artículo se organiza de la siguiente manera: primero, se discute la relación entre los derechos culturales y el derecho del patrimonio cultural. En segundo lugar, se analiza el marco de los derechos culturales de las comunidades patrimoniales, especialmente de las comunidades indígenas. En la tercera parte, se discuten los recientes desarrollos en el derecho internacional para el reconocimiento de un derecho al patrimonio como un derecho humano. Por último, se exploran algunas limitaciones

15 Giaccardi (2012), p. 2. 
en la participación de las comunidades en el actual modelo internacional de protección al patrimonio cultural. Las conclusiones resaltan el traslado hacia las comunidades locales del papel preponderante que tradicionalmente ha tenido el Estado-nación en las políticas patrimoniales.

\section{Derechos culturales y derecho del patrimonio CUltural}

El derecho cultural es un campo amplio que establece la cultura como objeto del derecho y regula, entre otras cosas, la propiedad, la protección, la circulación, la promoción y el uso de recursos culturales ${ }^{16}$. El derecho cultural abarca diferentes áreas jurídicas especializadas, como el derecho del arte, el derecho del deporte, los derechos de autor y la protección legal a los conocimientos tradicionales ${ }^{17}$. De manera que el derecho patrimonial o del patrimonio cultural se refiere a un régimen jurídico para la regulación de los recursos culturales constitutivos de la identidad de los grupos sociales ${ }^{18}$.

Se entienden por derechos culturales aquellos derechos relacionados con la cultura reconocidos en los diversos instrumentos internacionales de derechos humanos. La Declaración Universal de Derechos Humanos de 1948 reconoció los derechos a la educación ${ }^{19}$, a tomar parte libremente en la vida cultural de la comunidad, a gozar de las artes, a participar en el progreso científico y en los beneficios que de él resulten, y el derecho de los autores a la protección de los intereses morales y materiales sobre sus obras científicas, literarias o artísticas ${ }^{20}$. Además de estos derechos, el Pacto Internacional de Derechos Económicos, Sociales y Culturales de 1966 incluye, dentro del derecho a los beneficios de la cultura, la obligación de los Estados a respetar la libertad en la investigación científica y la actividad creadora ${ }^{21}$.

16 Nafziger, Paterson y Renteln (2010), p. 64.

17 NafZiger y Janis (2006), p. 317.

18 El derecho del patrimonio cultural como un área de estudio específica dentro de las ciencias jurídicas comenzó con la Convención de La Haya de 1954 para la Protección de los Bienes Culturales en caso de Conflicto Armado.

19 Declaración Universal de los Derechos Humanos (1948), art. 26.

20 Declaración Universal de los Derechos Humanos (1948), art. 27.

21 Pacto Internacional de Derechos Económicos, Sociales y Culturales (1966), art. 15; de igual forma, el Protocolo Adicional a la Convención Americana sobre Derechos Humanos en Materia de Derechos Económicos, Sociales y Culturales (1988), art. 14. 
Uno de los mayores obstáculos que ha impedido el desarrollo y discusión de los derechos culturales es que múltiples Estados observaron en estos derechos una posible amenaza para sus proyectos de consolidar una identidad nacional y un

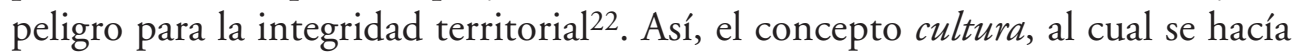
referencia durante la redacción de los primeros instrumentos internacionales era de alta cultura, es decir, lo relacionado con categorías de arte, piezas maestras y otras expresiones vinculadas a elites de tradición europea. En la década del 70, la interpretación del término cultura, en el marco de los derechos humanos, se expandió incorporando una noción no elitista que incluyó la cultura popular y diversas formas de vida ${ }^{23}$.

De manera simultánea, desde la segunda mitad del siglo XX, la Organización de las Naciones Unidas para la Educación, la Ciencia y la Cultura (Unesco) ha sido la principal organización en promover marcos internacionales para la protección del patrimonio cultural. La Unesco creó dos convenciones para luchar contra la importación, la exportación y la transferencia ilícitas de bienes culturales y la convención de 1972 para la protección del patrimonio mundial. Sin embargo, durante el siglo XX, el análisis del derecho del patrimonio cultural estuvo desconectado de los derechos culturales establecidos en los instrumentos de derechos humanos.

La ubicación del patrimonio cultural en el marco de los derechos humanos es parte de un reconocimiento muy reciente y en evolución de los derechos culturales. Aunque a nivel internacional los derechos culturales han sido catalogados como de tercera generación y llamados "la cenicienta" de los derechos humanos $^{24}$, cada día existe un mayor consenso sobre la importancia de los derechos culturales para el pleno goce de la dignidad humana ${ }^{25}$. Dentro de este contexto, recientes instrumentos jurídicos han reconocido el derecho al patrimonio y su conexión con los derechos humanos, especialmente a través de la participación de comunidades en los procesos de gestión patrimonial.

\footnotetext{
22 Stamatopoulou (2007), p. 5.

23 CHOW (2014).

24 Donders y Volodin (2007).

25 "Los derechos culturales son parte integrante de los derechos humanos, que son universales, indisociables e interdependientes. El desarrollo de una diversidad creativa exige la plena realización de los derechos culturales, tal como los definen el artículo 27 de la Declaración Universal de Derechos Humanos y los artículos 13 y 15 del Pacto Internacional de Derechos Económicos, Sociales y Culturales”. UnEsCo (2001), art. 5.
} 


\section{Derechos CUlturales y COMUNidAdes Patrimoniales}

El patrimonio cultural, al regular recursos de la identidad de las comunidades, está conectado con todos los derechos culturales ${ }^{26}$. La interrelación, la indivisibilidad y la interdependencia de los derechos humanos vinculan directamente el ejercicio de un derecho al patrimonio cultural con el derecho a participar en la vida cultural, con los derechos de autor ${ }^{27}$, los derechos políticos, la libertad de expresión, la libertad de creencias y de religión, el derecho a la información, los derechos lingüísticos, el derecho a la educación y los derechos de pueblos originarios.

En particular, los pueblos indígenas han reformado la comprensión de las funciones y la gestión del patrimonio, principalmente en sociedades que reconocen el multiculturalismo. El significado contemporáneo de la cultura en el derecho internacional está relacionado con las memorias colectivas que se conectan con las reivindicaciones de los pueblos indígenas ${ }^{28}$ y las ideas de justicia histórica ${ }^{29}$.

En el campo de los derechos de los pueblos indígenas y de lo que se ha denominado minorías nacionales ${ }^{30}$ existen derechos culturales que definen un marco que promueve las diferencias culturales al interior de los Estados. El Pacto Internacional de Derechos Civiles y Políticos de 1966 reconoció el derecho de libre determinación de todos los pueblos incluyendo su desarrollo cultural ${ }^{31}$. Así mismo, reconoció el derecho de las personas que pertenecen a comunidades étnicas, religiosas y lingüísticas a tener su propia vida cultural, a profesar y practicar su propia religión, y a emplear su propio idioma ${ }^{32}$.

26 LANGField et al. (2010).

27 Austin y Helfer (2011), p. 189.

28 CHOw (2014).

29 SHAHEED (2014).

30 La primera definición legal de minorías nacionales, dentro de un instrumento internacional, la estableció el Instrumento para la Protección de los Derechos de las Minorías de la Iniciativa de Europa Central (1994). En su artículo 1 se define como: "un grupo que es menor en número que el resto de la población del Estado, cuyos miembros siendo nacionales del Estado, poseen características étnicas, religiosas o lingüísticas diferentes al del resto de la población, y están guiados por el propósito de salvaguardar su cultura, tradiciones, religión o lenguaje". Una de las limitaciones de la definición es que está restringida al estatus de ciudadanía, lo cual deja desprotegidas a poblaciones migrantes, desplazadas, refugiadas, indocumentadas, entre otras.

31 Pacto Internacional de Derechos Civiles y Políticos (1966), art. 1.

32 Pacto Internacional de Derechos Civiles y Políticos (1966), art. 27. 
El Convenio 169 de la OIT sobre Pueblos Indígenas y Tribales es el principal instrumento jurídicamente vinculante que reconoce el derecho de las comunidades indígenas a participar y decidir sobre el uso y la gobernanza de su patrimonio cultural ${ }^{33}$. Dicho convenio estableció múltiples obligaciones para los Estados en el marco de los derechos culturales, especialmente las siguientes:

- Promover la plena efectividad de los derechos culturales de esas comunidades, respetando su identidad social y cultural, sus costumbres y tradiciones, y sus instituciones ${ }^{34}$.

- Adoptar medidas especiales para salvaguardar las personas, las instituciones, los bienes, el trabajo, las culturas y el medio ambiente de estos pueblos 35 .

- Consultar a las comunidades cada vez que se prevean medidas legislativas o administrativas susceptibles de afectarles directamente ${ }^{36}$.

- Garantizar el derecho de las comunidades a decidir sobre su propio proceso de desarrollo cultural ${ }^{37}$.

- Garantizar el derecho de conservar costumbres e instituciones propias, siempre que estas no sean incompatibles con los derechos fundamentales definidos por el sistema jurídico nacional ni con los Derechos humanos internacionalmente reconocidos ${ }^{38}$.

- Respetar la relación importante y especial que tienen las culturas con los territorios y sus valores espirituales ${ }^{39}$.

- Reconocer y promover la artesanía, las industrias rurales y comunitarias, y las actividades tradicionales relacionadas con la economía de subsistencia, como factores importantes del mantenimiento de la cultura de las comunidades y de su autosuficiencia y desarrollo económico ${ }^{40}$.

33 Foblets y Yassari (2013).

34 Convenio 169 de la OIT sobre Pueblos Indigenas y Tribales en Paises Independientes (1989), art. 2.

35 Convenio 169 de la OIT sobre Pueblos Indigenas y Tribales en Paises Independientes (1989), art. 4.

36 Convenio 169 de la OIT sobre Pueblos Indígenas y Tribales en Paises Independientes (1989), art. 6. En Colombia, el derecho a la consulta previa establecido en el Convenio 169 también aplica a comunidades afrodescendientes, el cual ha tenido un amplio desarrollo jurisprudencial por la Corte Constitucional.

37 Convenio 169 de la OIT sobre Pueblos Indigenas y Tribales en Paises Independientes (1989), art. 7.

38 Convenio 169 de la OIT sobre Pueblos Indigenas y Tribales en Paises Independientes (1989), art. 8.

39 Convenio 169 de la OIT sobre Pueblos Indígenas y Tribales en Países Independientes (1989), art. 13.

40 Convenio 169 de la OIT sobre Pueblos Indígenas y Tribales en Países Independientes (1989), art. 23. 
Otra referencia importante de los derechos culturales y su conexión con el patrimonio se encuentra en la Declaración de las Naciones Unidas sobre los Derechos de los Pueblos Indígenas, la cual reconoció el derecho que tienen estas comunidades:

"A mantener, controlar, proteger y desarrollar su patrimonio cultural, sus conocimientos tradicionales, sus expresiones culturales tradicionales y las manifestaciones de sus ciencias, tecnologías y culturas, comprendidos los recursos humanos y genéticos, las semillas, las medicinas, el conocimiento de las propiedades de la fauna y la flora, las tradiciones orales, las literaturas, los diseños, los deportes y juegos tradicionales, y las artes visuales e interpretativas" 41 .

Aunque la declaración sobre los derechos de los pueblos indígenas no es vinculante, tiene un efecto legal y político que empodera a las comunidades indígenas para defender su derecho al patrimonio cultural. Uno de los ejes centrales de la declaración son los derechos culturales y las disposiciones de la declaración son tenidas en cuenta en el Examen Periódico Universal (EPU) que realiza el Consejo de Derechos Humanos de las Naciones Unidas.

En el ámbito de la protección de los derechos de las comunidades indígenas, las cortes internacionales han defendido el derecho al patrimonio. La Corte Interamericana de Derechos humanos $(\mathrm{CIDH})$ ha resaltado la importancia del patrimonio cultural dentro de una perspectiva holística, en la cual la defensa de otros bienes jurídicos, como el derecho a la propiedad y a la tierra, está íntimamente relacionada con la salvaguardia de diferentes formas de vida cultural. Así, por ejemplo, la Corte ha manifestado que:

"Para las comunidades indígenas la relación con la tierra no es meramente una cuestión de posesión y producción sino un elemento material y espiritual del que deben gozar plenamente, inclusive para preservar su legado cultural y transmitirlo a las generaciones futuras" ${ }^{\prime 2}$.

Aquí la mención de la Corte al legado cultural y la transmisión intergeneracional nos remite a la noción de patrimonio. Sin embargo, en este escenario, la protección al patrimonio e identidad cultural surge de manera indirecta al proteger otros derechos.

41 United Nations (2007), art. 31.

42 Corte Interamericana de Derechos Humanos, Comunidad Mayagna (Sumo) Awas Tingni con Nicaragua, 31 de agosto de 2001 . 
De manera similar, la Corte Africana de Derechos Humanos y de los Pueblos, en la icónica decisión que defiende los derechos de la comunidad indígena Ogiek de Kenia (caso Endorois), reconoció el derecho al patrimonio cultural de manera indirecta; está conectado, entre otros, al derecho al desarrollo, a la tierra y a las formas de vida de la comunidad indígena. La Corte africana fue explícita en reconocer el derecho a la protección del patrimonio cultural como parte de los derechos culturales:

"La protección del derecho a la cultura va más allá del deber, de no destruir o deliberadamente debilitar a los grupos minoritarios, sino que obliga al respeto y protección de su patrimonio cultural, esencial para la identidad del grupo. A este respecto, la cultura debe interpretarse en su sentido más amplio, abarcando la forma total de vida de un grupo en particular, incluyendo las lenguas del grupo, símbolos como los códigos de vestir [...] y valores compartidos de sus miembros que reflejan su distintivo carácter y personalidad” 43 .

Algunas cortes nacionales, como la Corte Constitucional de Colombia, han protegido el derecho fundamental a la integridad cultural de las comunidades indígenas ${ }^{44}$. Una consecuencia fundamental de interpretar el derecho al patrimonio cultural, como un derecho humano, es que su protección sería amparable de manera autónoma y directa.

Un reconocimiento explícito al derecho al patrimonio y el derecho de las comunidades a participar en la gestión patrimonial se da en el contexto de instrumentos internacionales del siglo XXI que analizaremos en la siguiente sección.

\section{Desarrollos recientes del derecho al patrimonio}

CULTURAL COMO UN DERECHO HUMANO

El derecho internacional del patrimonio cultural ha evolucionado drásticamente en las últimas dos décadas, ha establecido nuevas formas de protección y ha adquirido nuevas funciones. Las comunidades, en estos nuevos desarrollos de las políticas y la gestión patrimonial, reemplazan y/o complementan el tradicional papel de los expertos, especialmente porque las políticas patrimoniales

43 Corte Africana de Derechos Humanos y de los Pueblos, African Commission on Human and Peoples' Rights con Republic of Kenya, 26 de mayo de 2017.

44 Por ejemplo, ver las sentencias: Corte Constitucional de Colombia, rol No SU-510, de 18 de septiembre de 1998, y Corte Constitucional de Colombia, rol No T-1022, de 20 de septiembre de 2001. 
están trayendo a su alcance de salvaguardia una amplia gama de nuevos objetos, como el patrimonio inmaterial, el patrimonio documental y una visión más compleja de los derechos culturales que incluye el derecho al acceso y disfrute del patrimonio cultural.

\subsection{Patrimonio cultural inmaterial}

En 2003, la Unesco adoptó la Convención para la Salvaguardia del Patrimonio Cultural Inmaterial (PCI). Esta convención marca un importante cambio en el entendimiento del patrimonio. La convención del PCI es el resultado de la mayor participación de Asia y países del sur global en las políticas de la Unesco. Estos Estados llamaron la atención sobre las reivindicaciones para una interpretación del patrimonio más inclusiva, con énfasis especial en la protección de las tradiciones orales, el patrimonio de los pueblos originarios y un acercamiento a procesos patrimoniales más incluyentes con la participación de las comunidades. La convención define el patrimonio cultural inmaterial de la siguiente manera:

"[son] los usos, representaciones, expresiones, conocimientos y técnicas -junto con los instrumentos, objetos, artefactos y espacios culturales que les son inherentes-que las comunidades, los grupos y en algunos casos los individuos reconozcan como parte integrante de su patrimonio cultural. Este patrimonio cultural inmaterial, que se transmite de generación en generación, es recreado constantemente por las comunidades y grupos en función de su entorno, su interacción con la naturaleza y su historia, infundiéndoles un sentimiento de identidad y continuidad y contribuyendo así a promover el respeto de la diversidad cultural y la creatividad humana" ${ }^{45}$.

La Convención del PCI destaca que la mundialización está creando peligros para el patrimonio inmaterial, produciendo "graves riesgos de deterioro, desaparición y destrucción del patrimonio cultural inmaterial, debido en particular a la falta de recursos para salvaguardarlo" 46 . Los procesos de desarrollo económico, modernización y de explotación de recursos naturales son agentes centrales en la desaparición del patrimonio cultural inmaterial ${ }^{47}$. Especialmente, el patrimonio

45 Convención para la Salvaguardia del Patrimonio Cultural Inmaterial (2003), artículo 2.

46 Convención para la Salvaguardia del Patrimonio Cultural Inmaterial (2003), preámbulo.

47 Por ejemplo, el crecimiento económico de Mongolia, debido a las actividades mineras, está transformando drásticamente el modo de vida de la mayor parte de su población. Mongolia es el segundo país con el número más alto de inscripciones en la lista de la Unesco del patrimonio inmaterial en peligro, lo 
e identidad cultural que se encuentra en mayor riesgo es el de las comunidades que históricamente han sido marginalizadas por el Estado-nación ${ }^{48}$.

La participación de las comunidades es un aspecto clave del patrimonio inmaterial ${ }^{49}$. Este es un enfoque local que difiere de la orientación universalista de la Convención de la Unesco de 1972. Por ejemplo, las comunidades de origen deben ser consultadas para la nominación e inscripción de su patrimonio inmaterial en la lista de la Unesco ${ }^{50}$. Este nuevo enfoque al patrimonio mundial en el Convenio del PCI reconoce los valores locales y la participación comunitaria ${ }^{51}$.

Sin embargo, lo que se pueda designar oficialmente como patrimonio está limitado por la visión universal de los derechos humanos ${ }^{52}$. La actual relatora especial sobre los derechos culturales, Karima Bennoune, ha sido enfática en afirmar que "cuando hay prácticas que vulneran los Derechos humanos, el derecho de los Derechos humanos a veces obliga a que se produzca un cambio cultural [...]"53. Las expresiones culturales protegidas por el derecho del patrimonio cultural se encuentran delineadas por los valores expresados, explicita e implícitamente, en el sistema universal de derechos humanos ${ }^{54}$. Las costumbres que entran en

cual se debe al rápido cambio social que atraviesa ese país a través de la urbanización, las tecnologías de la información, el capitalismo y la globalización económica y cultural. The Economist (2012); UNESCO (2013).

48 Por ejemplo, el patrimonio lingüístico de las comunidades étnicas y de grupos subalternos se encuentra en grave peligro a nivel mundial. Se estima que, de las aproximadamente 6000 lenguas que existen actualmente en el mundo, para finales de este siglo, alrededor del $90 \%$ habrá desaparecido. KraUSS (2007), p. 9.

49 Convención para la Salvaguardia del Patrimonio Cultural Inmaterial (2003), artículo 15.

50 UnesCo (2014).

51 Esto ocurre a pesar de que existen múltiples tensiones entre los Estados-nación, el sistema internacional de la Unesco y las comunidades patrimoniales, respecto a las prácticas burocráticas que la convención y su implementación crean. Ver Hafstein (2014).

52 El artículo 2 de la Convención del PCI expresa que "se tendrá en cuenta únicamente el patrimonio cultural inmaterial que sea compatible con los instrumentos internacionales de derechos humanos existentes y con los imperativos de respeto mutuo entre comunidades, grupos e individuos y de desarrollo sostenible". De manera similar, el artículo 5 de la Declaración y Programa de Acción de Viena (1993) advierte que "Debe tenerse en cuenta la importancia de las particularidades nacionales y regionales, así como de los diversos patrimonios históricos, culturales y religiosos, pero los Estados tienen el deber, sean cuales fueren sus sistemas políticos, económicos y culturales, de promover y proteger todos los derechos humanos y las libertades fundamentales".

53 Bennoune (2016).

54 Este es un debate complejo relacionado con el relativismo cultural o universalismo de los derechos humanos. Para una discusión general del tema ver Roy y Annicchino (2013). 
conflicto con los derechos humanos no pueden ser protegidas por las políticas del patrimonio cultural. Por ejemplo, los Estados tienen la obligación de abolir las prácticas tradicionales que sean perjudiciales para la salud de los niños ${ }^{55}$.

Bajo esta óptica, el derecho patrimonial debe estar presente como herramienta de la gestión del cambio, servir para abrir diálogos y empoderar a las comunidades para lograr sus propias transformaciones. Por ejemplo, bajo el prisma de los derechos humanos en Colombia, la práctica tradicional de algunas comunidades U'wa de entregar a los gemelos recién nacidos como alimento a las deidades ${ }^{56}$ o la ablación-mutilación genital femenina practicada por ciertas comunidades Emberá ${ }^{57}$ no pueden considerarse patrimonio cultural inmaterial. Bajo estas circunstancias, los derechos humanos se deben usar para promover transformaciones en las prácticas culturales, como de hecho está sucediendo con la transformación de las practicas mencionadas de las comunidades U’wa y Emberá.

\subsection{Derecho de acceso y disfrute del patrimonio cultural}

El acceso y disfrute del patrimonio cultural, como derecho humano, comprende individuos y comunidades patrimoniales que participan en la identificación, curación, interpretación y designación de los recursos culturales que desean heredar a las generaciones futuras ${ }^{58}$. El Convenio marco del Consejo de Europa sobre el valor del patrimonio cultural para la sociedad (Convenio de Faro) y el informe de la experta independiente en el campo de los derechos culturales para el Alto Comisionado de las Naciones Unidas para los Derechos Humanos (ACNUDH) son dos avances importantes en el reconocimiento de este derecho.

El Convenio marco del Consejo de Europa sobre el valor del patrimonio cultural para la sociedad (Convenio de Faro) del 2005, que entró en vigor en 2011, fue el primer instrumento internacional que reconoció explícitamente el vínculo entre el derecho al patrimonio cultural y los derechos humanos. El artículo primero del convenio establece que: "Los derechos relativos al patrimonio cultural son inherentes al derecho a tomar parte en la vida cultural, consagrado en

55 Convención sobre los Derechos del Niño (1989), art. 24.

56 SÁnChez de GuZMán (2006).

57 Henao y Pineda (2010).

58 Henao y Pineda (2010). Shaheed (2011), p. 15. 
la Declaración Universal de Derechos Humanos"59. Lo anterior reafirma que el patrimonio es esencial para participar en la vida cultural y el acceso y la utilización del patrimonio forman parte de los derechos culturales ${ }^{60}$. Esto implica derechos individuales y colectivos de acceso, interpretación y participación en la designación del patrimonio ${ }^{61}$.

El Convenio de Faro normativiza a nivel internacional las nuevas concepciones del patrimonio señalando el derecho al patrimonio cultural como parte de los derechos humanos. Este es un paso que invita a los gobiernos a establecer políticas adecuadas para hacer efectivo ese derecho. Para el año 2018, solo 18 países la habían ratificado. El abogado español Luis Pérez Prat resalta que España, Francia, Alemania y Reino Unido no hayan ratificado la Convención de Faro, y sugiere, a modo de pregunta, que la falta de ratificación podría ser por las obligaciones que los Estados asumen al reconocer el derecho al patrimonio cultural como derecho humano ${ }^{62}$. Sin embargo, el convenio no genera obligaciones directas en los Estados.

Como se mencionó anteriormente, una de las razones para el poco desarrollo de los derechos culturales es el miedo de los Estados a que estos derechos puedan promover el separatismo. En el contexto europeo actual, algunos Estados están confrontando movimientos independentistas, de manera que puede generar temor para la integridad y unidad del Estado-nación el reconocimiento de un derecho humano al patrimonio y el reconocimiento de múltiples comunidades ${ }^{63}$. Sin embargo, el Convenio de Faro tiene un enfoque que promueve, por medio del patrimonio, no las rupturas, sino el diálogo para crear democracias más activas, participativas y plurales. En ese sentido, el convenio hace hincapié en que los Estados deberán considerar la relación de cada comunidad patrimonial con los recursos patrimoniales que allí se identifican ${ }^{64}$. El enfoque de comunidades patrimoniales en el convenio de Faro es amplio; incluye refugiados, migrantes, minorías étnicas, culturales

59 Framework Convention on the Value of Cultural Heritage for Society (2005), art. 1.

60 Shaheed (2011); Meyer-Bisch (2009), p. 59.

61 DolfF-BoneKÄMPER (2009), p. 70.

62 Pérez-Prat (2014), p. 340.

63 El caso más notorio de recientes intentos de secesión en Europa es el movimiento separatista catalán en España.

64 Framework Convention on the Value of Cultural Heritage for Society (2005), art. 12 (b). 
y otros grupos políticos. Este nuevo enfoque reconoce el papel del patrimonio cultural en la promoción de la diversidad cultural.

Junto al Convenio de Faro se encuentra la interpretación del patrimonio cultural dentro de los derechos culturales por parte del Alto Comisionado de las Naciones Unidas para los Derechos Humanos (ACNUDH). En el 2009, el ACNUDH nombró a Farida Shaheed como experta independiente en el campo de los derechos culturales. Su segundo informe presentado al Consejo de Derechos Humanos en 2011 discutió el derecho de acceso y disfrute del patrimonio cultural como parte del Derecho Internacional de los Derechos Humanos ${ }^{65}$. Farida Shaheed concluyó que:

"El derecho de acceso al patrimonio cultural y de su disfrute incluye el derecho de las personas y las comunidades, entre otras cosas, a conocer, comprender, entrar, visitar, utilizar, mantener, intercambiar y desarrollar el patrimonio cultural, así como a beneficiarse del patrimonio cultural y de la creación de los otros. Incluye también el derecho a participar en la determinación, la interpretación y el desarrollo del patrimonio cultural, así como de diseñar y ejecutar políticas y programas de preservación y salvaguardia" 66 .

Este derecho de acceso y disfrute que plantea Farida Shaheed se conecta directamente con el funcionamiento de las políticas y programas de gestión patrimonial, lo cual incluye el derecho de las comunidades a participar en los procesos de selección, nominación, designación del patrimonio cultural. Esto genera una ruptura con el tradicional enfoque jerárquico administrado de manera exclusiva por el Estado. Esta nueva perspectiva ha generado la posibilidad de que los recursos culturales que dan cuenta de violaciones de derechos humanos por parte del Estado se conviertan en patrimonio.

\subsection{Patrimonio documental y victimas de violaciones de los derechos humanos}

La patrimonialización, en el contexto contemporáneo de los derechos humanos, tiene el potencial de servir en la creación de procesos que empoderen a los que han sufrido opresiones para así revelar y desafiar las injusticias. El uso contemporáneo del patrimonio cultural está llamado a conectarse con la justicia histórica y con el ejercicio de diferentes derechos humanos emergentes, como el

65 SHAHEED (2011).

66 Shaheed (2011), p. 21. 
derecho a la verdad y el derecho a la memoria. La socióloga y feminista Farida Shaheed destaca lo siguiente:

"El patrimonio cultural no está limitado a objetos y manifestaciones de los que las personas y las comunidades pueden estar orgullosas. En algunos casos, el patrimonio recuerda los errores cometidos en el pasado y las acciones que reflejan el lado más oscuro de la humanidad, cuya memoria también debe ser transmitida a las futuras generaciones, aunque de una manera diferente" 67 .

Los procesos de patrimonializacion de esta clase de recursos culturales cobran mayor importancia cuando han sido los Estados los responsables de las violaciones de derechos humanos.

El patrimonio documental de la humanidad presenta buenos ejemplos de la patrimonialización de recursos culturales relacionados con comunidades que han sido víctimas de graves violaciones de derechos humanos. El proyecto Memoria del Mundo de la Unesco ha incorporado dentro de sus procesos de designación la evaluación de la importancia social y espiritual de los documentos para sus comunidades, como un criterio al momento de evaluar la inclusión de una nominación en el registro de Memoria del Mundo. La importancia social y espiritual de un documento representa su valor emocional para una comunidad por la forma en que contribuye a su identidad y a la cohesión social. Dentro de los archivos que se han registrado y convertido en patrimonio de la humanidad se encuentran documentos que representan las injusticias históricas que comunidades indígenas han sufrido. Especialmente Australia y Nueva Zelanda han sido líderes en este enfoque.

Nueva Zelanda logró incluir, dentro del proyecto de Memoria del Mundo, documentos de gran significancia política para las actuales reparaciones económicas y culturales que las comunidades indígenas han estado negociando con el gobierno neozelandés. El Tratado de Waitangi, firmado en 1840 por múltiples jefes de comunidades maorí y representantes de la corona inglesa, ha sido declarado por la Unesco como patrimonio de la humanidad. La sistemática violación de los derechos de los maoríes durante el siglo XIX y parte del siglo XX y el incumplimiento del Tratado de Waitangi ha dado lugar a un proceso de

67 Shaheed (2011), p. 5. 
justicia histórica ${ }^{68}$. Este documento declarado patrimonio del mundo es el pilar fundamental para la reparación de sus derechos.

La interpretación y la investigación del patrimonio que da cuenta de hechos atroces e injusticias históricas es importante para lograr reparaciones en medio de debates democráticos. La Unesco ha incorporado dentro de la memoria del mundo archivos que son testimonio de la violación de los derechos humanos de múltiples comunidades políticas, étnicas y culturales, como por ejemplo el Archivo del Museo del Genocidio de Tuol Sleng en Camboya, los Archivos del Terror de Paraguay y el Archivo de Derechos Humanos de Chile ${ }^{69}$. Estos patrimonios documentales son usados con múltiples propósitos, pueden ser parte del interés político de los gobiernos nacionales para fomentar una cultura de reconciliación, transmitir a las generaciones futuras las traumáticas experiencias de las generaciones recientes, servir como instrumento de reparación simbólica para las víctimas, reforzar la protección jurídica y material de documentos que sirven como evidencias, fomentar la memoria colectiva, ser fuente para la investigación académica y servir como referencia para la no repetición.

Si bien existen avances importantes en el reconocimiento del derecho humano al patrimonio cultural, hay que señalar que el sistema del derecho del patrimonio aún permanece anclado en un modelo de gobernanza diseñado en el siglo XX. A continuación, examinaremos dos de los principales retos del sistema internacional del patrimonio cultural que requieren actualización para incorporar un enfoque compatible con los derechos humanos de las comunidades.

\section{Principales Retos Para incorporar a las COMUNidADES}

EN EL ACTUAL SISTEMA INTERNACIONAL DEL PATRIMONIO CULTURAL

En el siglo XX se construyó un modelo de derecho del patrimonio cultural basado en unas posturas binarias entre universalismo y nacionalismo ${ }^{70}$. Las

68 HaYward y Wheen (2004).

69 Chile fue el primer país latinoamericano en utilizar el sistema de patrimonio documental mundial para representar sus transiciones en la identidad política y cultural después de un período de dictadura entre 1973 y 1990. En el año 2003, la Unesco designó el Archivo de Derechos Humanos de Chile como parte de la Memoria del Mundo. En la nominación para la designación patrimonial, el Archivo Nacional de Chile resaltó la utilidad de la inclusión en el registro mundial de la Unesco para una comprensión del patrimonio como decisión política del presente para la construcción del futuro. La patrimonialización del Archivo de Derechos Humanos de Chile sirve como artefacto simbólico que hace parte de las políticas de la memoria que conjugan el plano nacional e internacional. Chile (2003).

70 Merryman (1986), p. 831. 
convenciones y otros instrumentos jurídicos establecidos durante el siglo pasado son el marco fundamental del actual sistema mundial de patrimonio. La repatriación de bienes culturales y los procesos de patrimonialización en la lista mundial de la Unesco no cuentan con la participación de comunidades. Incluir a las comunidades por medio de los derechos humanos en el funcionamiento y operación de estos procesos es necesario para deconstruir el modelo binario entre universalismo y nacionalismo, y dar lugar a lo local como eje fundamental de la gestión patrimonial.

\subsection{Tráfico de bienes culturales y repatriación}

En el plano internacional y local, los objetos culturales de las comunidades indígenas fueron usados por los gobiernos para adelantar un discurso sobre un patrimonio cultural perteneciente al Estado-nación. Sin embargo, las comunidades indígenas no fueron tenidas en cuenta en el diseño de esos instrumentos. En 1960, durante la conferencia general de la Unesco, México y Perú propusieron establecer medidas internacionales para luchar contra el tráfico de bienes culturales, especialmente objetos precolombinos. Simultáneamente a las negociaciones multilaterales, México y los Estados Unidos firmaron un tratado bilateral de cooperación para la recuperación y devolución de las propiedades arqueológicas, históricas y culturales saqueadas ${ }^{71}$. Los países del sur global utilizaron los foros internacionales en un intento por detener el asalto a sus recursos culturales. Estas propuestas se produjeron en un contexto de álgidas políticas nacionalistas de los Estados poscoloniales ${ }^{72}$.

En 1970, la Unesco adoptó la Convención sobre las Medidas para Prohibir e Impedir la Importación, Exportación y Transferencia de Propiedad Ilícitas de Bienes Culturales ${ }^{73}$. La convención obliga a los Estados partes a establecer medidas legislativas contra el comercio ilícito de bienes culturales ${ }^{74}$. Los con-

\footnotetext{
71 JOWERS (2003).

72 La propuesta de México y Perú coincide con la declaración de la ONU en 1960 sobre la concesión de la independencia a los países y pueblos coloniales, ver РROTt (2012).

73 Convención sobre las Medidas que deben Adoptarse para Prohibir e Impedir la Importación, la Exportación y la Transferencia de Propiedad Ilícitas de Bienes Culturales (1970). Sobre este mismo tema también es importante resaltar el Convenio de Unidroit sobre los Bienes Culturales Robados o Exportados Ilícitamente (1995).
}

74 Para un análisis detallado ver O’Keefe (2007). 
ceptos de importación y exportación están sujetos al ámbito territorial nacional gobernado por los Estados. Este criterio no tiene en cuenta que las comunidades patrimoniales se pueden ubicar dentro del territorio de múltiples Estados ${ }^{75}$, lo cual ha derivado conflictos entre diferentes Estados o entre las comunidades y los Estados $^{76}$.

Además, son los Estados quienes tienen la potestad de seleccionar los objetos que serán protegidos como patrimonio cultural. Esta visión menoscaba los derechos de comunidades indígenas, a quienes no se les reconoce el derecho de controlar la salida de objetos culturales de sus territorios, ni la garantía de participar a nivel nacional en los procesos de designación patrimonial sobre sus recursos. Además, en la práctica, las comunidades de origen no son tenidas en cuenta para decidir acerca de los recursos sobre los cuales los Estados logran restitución ${ }^{77}$.

El alcance de esta convención es también limitado frente a las necesidades y reclamaciones actuales de múltiples comunidades, cuyos objetos culturales fueron trasladados durante tiempos coloniales. De gran resonancia es el caso de la repatriación de restos humanos de comunidades indígenas que se encuentran en museos europeos. Por ejemplo, en las 2007 comunidades indígenas Maorí de Nueva Zelanda, después de años de esfuerzos, lograron el retorno de cabezas de sus antepasados que se encontraban en el museo Rouen en Francia ${ }^{78}$. La repatriación patrimonial se encuentra en esos casos sujeta a negociaciones políticas y consideraciones éticas que están relacionadas con los derechos humanos. La repatriación es, en esos contextos, un acto de buena voluntad de los Estados al no existir instrumentos jurídicos necesarios para realizar exigencias legales.

\subsection{Patrimonialización o cultural y natural mundial}

La Convención de la Unesco de 1972 estableció el criterio del valor universal excepcional (VUE) para la selección de aquellos recursos que podrían

\footnotetext{
75 Por ejemplo, las comunidades indígenas Sami se encuentran ubicadas en territorios transfronterizos que abarcan partes de Noruega, Suecia, Finlandia y Rusia.

76 Esta clase de situación ha sido frecuente cuando convergen temas de propiedad intelectual y patrimonio cultural, por ejemplo, en el sudeste asiático con los tradicionales diseños de batik.

77 ОСНOA (2012), p. 142.

78 Bioy (2011).
} 
ser designados como patrimonio mundial ${ }^{79}$. La Unesco define el VUE como la "importancia cultural y/o natural tan extraordinaria que trasciende las fronteras nacionales y cobra importancia para las generaciones presentes y venideras de toda la humanidad" 80 . El criterio del VUE refleja un enfoque global y universalista del patrimonio. El VUE no corresponde necesariamente con los valores que las comunidades locales asignan al patrimonio ${ }^{81}$.

En 1980, como parte de la implementación de la Convención de 1972, la Unesco creó la Lista del Patrimonio Mundial ${ }^{82}$. La lista consta de edificios históricos, monumentos, ciudades, caminos patrimoniales, paisajes culturales y áreas naturales que demuestran un valor universal excepcional. Para que un recurso cultural ingrese en la lista requiere que los Estados presenten candidaturas que luego son procesadas por la Unesco y evaluadas por un grupo de expertos. El comité del patrimonio mundial llega a una decisión que designa las entradas de la lista.

El sistema internacional del patrimonio cultural involucra la gestión del patrimonio en tres diferentes niveles, el global por las organizaciones internacionales, el nacional por los Estados y el local por las comunidades. El Estado-nación ha sido el actor principal en el sistema patrimonial al centralizar el poder de los tres diferentes niveles de gobernanza del patrimonio. Una de las motivaciones de los Estados para invertir el capital necesario para la inscripción en el patrimonio mundial son los réditos económicos que se pueden derivar en campos como la especulación inmobiliaria y el turismo.

Para comienzos del año 2017, la Lista del Patrimonio Mundial contenía 1052 inscripciones, de las cuales el $47 \%$ correspondían a bienes culturales y naturales de Europa y Norteamérica ${ }^{83}$. Esta sobrerrepresentación va de la mano con el VUE que, aunque pretende ser neutral y universal, tiene afinidad con los valores del norte global. Además, la sobrerrepresentación demuestra la capacidad de estos países en la gestión del sistema patrimonial a nivel internacional, lo cual

\footnotetext{
79 Convención sobre la Protección del Patrimonio Mundial Cultural y Natural (1972), art 1.

80 UnesCo (2008).

81 Para un análisis detallado del valor universal excepcional ver LABADI (2013); Chenevez y Novello (2015).

82 Valderrama (1995), p. 85.

$83 \mathrm{http}: / /$ whc.unesco.org/en/list/stat\#d1
} 
requiere de capital económico, intelectual y relacional ${ }^{84}$. Aunque la preservación de los sitios del patrimonio mundial es de interés de la comunidad internacional, la responsabilidad directa recae en los Estados y son las comunidades locales quienes en algunos casos se benefician o en otros se ven afectadas directamente con la patrimonialización ${ }^{85}$.

La patrimonialización tiende a generar cambios económicos y culturales acelerados y desequilibrados alrededor de los recursos que hacen parte del patrimonio mundial, como la sustitución de producción artesanal por servicios industriales y turísticos, procesos de gentrificación y desaparición precipitada de elementos claves de la identidad ${ }^{86}$. Estos fenómenos pueden impactar de manera negativa los derechos humanos de las comunidades locales. Los derechos y el bienestar de comunidades locales, especialmente cuando constituyen comunidades vulnerables frente al poder del Estado o del interés de elites locales y transnacionales, no son evaluados de forma anterior al proceso de designación patrimonial ni monitoreados de forma permanente ${ }^{87}$.

En las últimas dos décadas, el patrimonio de las comunidades indígenas se ha convertido en un protagonista de la Lista del Patrimonio Mundial. Los Estados están usando el sistema internacional del patrimonio para manifestar una imagen multicultural a nivel internacional. Por ejemplo, el Sistema de Carreteras Qhapaq Nan-Andina es un conjunto de caminos que comprende más de 30.000 kilómetros, fue construida por una cultura andina preinca hace más de 2000 años y alcanzó su punto máximo de expansión durante el gobierno inca en el siglo XVI ${ }^{88}$. Actualmente, los remanentes de Qhapaq Nan siguen en uso y algunas partes de la carretera han sido modernizadas. El gobierno peruano

84 En 1994, la Unesco lanzó una estrategia global para crear una lista más representativa, equilibrada y creíble. Sin embargo, para el año 2011, investigadores de la Universidad de Zurich encontraron que la estrategia no ha tenido efectos, al contrario, el desequilibrio ha aumentado, STEINER y FrEY (2011).

85 LABAdi y Long (2010).

86 Un ejemplo es Cartagena de Indias en Colombia. Ver http://www.tres.memoriasdelalibertad.org; Morales (2013).

87 Cada seis años los Estados son invitados por la Unesco a enviar un reporte periódico sobre el estado de conservación de los bienes declarados patrimonio mundial. Además, existe un sistema de monitoreo reactivo para aquellos bienes que se encuentren amenazados. Una preocupación por los derechos humanos está ausente en las discusiones sobre las estrategias, objetivos y mecanismos de monitoreo. Esta ausencia es evidente, por ejemplo, en las discusiones de Vicenza en el 2002, UnESCO e ICCROM (2004).

88 Hyslop (1984). 
tomó la iniciativa en 2001 para lograr la designación de Patrimonio Mundial para Qhapaq Nan ${ }^{89}$. En los años siguientes y con el apoyo de Argentina, Bolivia, Chile, Colombia y Ecuador, la ruta patrimonial fue nominada conjuntamente en la Unesco y finalmente designada en $2014^{90}$. Estos países se están moviendo hacia una representación política de Estados multiculturales donde el reconocimiento de los pueblos indígenas es parte de su política nacional. Sin embargo, estos gobiernos no involucraron a las comunidades como participantes reales en el proceso de toma de decisiones para este proyecto patrimonial ${ }^{91}$.

El Mecanismo de Expertos sobre los Derechos de los Pueblos Indígenas del Consejo de Derechos Humanos de las Naciones Unidas ha sido enfático y claro en recomendar la consulta y participación activa de las comunidades indígenas en el funcionamiento de las convenciones de la Unesco ${ }^{92}$. Esta recomendación aboga por el consentimiento previo, libre e informado por parte de las comunidades para que sus recursos culturales y naturales sean nominados y designados como patrimonio de la humanidad.

\section{Conclusión}

La inclusión de los derechos humanos dentro de los discursos y prácticas del patrimonio cultural ha promovido la desconcentración del poder de los Estados en los procesos de gestión patrimonial. Este cambio ha dado lugar a una participación cada vez más relevante de las comunidades patrimoniales, especialmente los pueblos indígenas. El derecho al patrimonio es un derecho autónomo, tanto de carácter individual como un derecho colectivo. Como derecho colectivo, se manifiesta de forma clara en el derecho de los pueblos indígenas a la libre determinación y en su derecho a mantener, controlar, proteger y desarrollar su identidad cultural.

En la práctica, la consulta a las comunidades en los procesos de selección y patrimonialización es deficiente y su participación sigue determinada por intereses y representaciones del Estado-nación. El sistema internacional del patrimonio administrado por la Unesco podría ser más riguroso, vigilante y exigente para

\footnotetext{
89 La capital del imperio Inca estuvo localizada en Cusco, actual territorio del Estado peruano.

90 UnEsCo (2014b), p. 240.

91 Jallade (2011); Korstanje y García (2007); Losson (2017).

92 Expert Mechanism Advice No 2 (2011). Indigenous peoples and the right to participate in decisionmaking.
} 
que las designaciones de objetos y prácticas reconocidos como patrimonio de la humanidad sean gestionadas bajo el prisma de los derechos humanos. En ese sentido, es necesario un mecanismo de monitoreo constante que dé cuenta de los impactos de la patrimonialización en los derechos humanos de las comunidades locales como requisito para que un objeto o una práctica mantenga el estatus de patrimonio de la humanidad.

El reconocimiento del derecho al acceso y disfrute del patrimonio cultural está en sus inicios. Su desarrollo para promover las diferencias culturales y las políticas multiculturales dependerá no solo del continuo empoderamiento de los pueblos indígenas, sino también del reconocimiento de otras comunidades patrimoniales, como los inmigrantes y refugiados, que son parte esencial de los actuales procesos de globalización.

BibLIOGRAFÍA

Austin, Graeme y Helfer, Laurence (2011). Human Rights and Intellectual Property-Mapping the Global Interface (Cambridge, Cambridge University Press).

Bennoune, Karima (2016). Informe de la Relatora Especial sobre los Derechos Culturales, Destrucción Intencional del Patrimonio Cultural (Naciones Unidas, A/71/317).

Bioy, Xavier (2011). "Le Statut des Restes Humains", Archéologiques, Revue du droit public et de la science politique en France et à l'Étranger, Vol. 1, (No 1), pp. 89-111.

Chenevez, Alain y Paglianti, Nanta Novello (2015). Linvention de la Valeur Universelle Exceptionnelle de l'Unesco: une Utopie Contemporaine (París, L'Harmattan).

CHILE (2003). Nomination of the Human Rights Archive of Chile to the MOW Register (Unesco).

Chow, Pok Yin S. (2014). "Culture as Collective Memories: An Emerging Concept in International Law and Discourse on Cultural Rights", en Human Rights Law Review, Vol. 14 (No 4), pp. 611-646.

Cornu, Marie (2008). "Notions Juridiques du Patrimoine et de la Création: les Entrelacs", Revue Générale de Droit, Vol. 38, No 2, pp. 281-302.

DolfF-BoneKÄmper, Gabi (2009). "The Social and Spatial Frameworks of Heritage - What Is New in the Faro Convention?", en PALMER, Robert, Heritage and beyond (Strasbourg, Council of Europe), pp. 69-74. 
Donders, Yvonne y Volodin, Vladimir (2007). Human Rights in Education, Science, and Culture: Legal Developments and Challenges (París, Unesco/ Ashgate).

Fairclough, Graham (2009). "New heritage frontiers", en Palmer, Robert, Heritage and beyond (Strasbourg, Council of Europe), pp. 29-42.

Figueroa Yáñez, Gonzalo (1997). El Patrimonio (Santiago, Editorial Jurídica de Chile).

Foblets, Marie-Claire y Yassari, Nadjma (2013). Legal Approaches to Cultural Diversity/Approches Juridiques de la Diversité Culturelle (The Hague, Martinus Nijhoff Publishers).

GiACCARDI, Elisa (2012). "Introduction: Reframing Heritage in a Participatory Culture", en Giaccardi, Elisa (edit.), Heritage and Social Media Understanding Heritage in a Participatory Culture (New York, Routledge).

Graham, Brian (2002). "Heritage as Knowledge: Capital or Culture?", Urban Studies, Vol. 39 (No 5-6), pp. 1003-1017.

Hafstein, Valdimar Tr. (2014). "Protection as Dispossession: Government in the Vernacular", en Kapchan, Deborah (edit.), Cultural Heritage in Transit Intangible Rights as Human Rights (Philadelphia, University of Pennsylvania Press).

HaYWARD, Janine y WheEn, Nicola (2004) (edit.). The Waitangi Tribunal: Te Roopu Whakamana i te Tiriti o Waitangi (Wellington, Bridget Williams Books).

Henao, Juanita y PIneda, Claudia (2010). El Proyecto Embera Wera: Una experiencia de cambio cultural para la eliminación de la ablación genital femenina y la promoción de los derechos de mujeres Embera de los municipios de Mistrató y Pueblo Rico del Departamento de Risaralda (Bogotá, ICBF, Programa Integral contra las Violencias de Género del F-ODM, Fondo de Población de las Naciones Unidas).

Hyslop, John (1984). The Inca Road System (New York, Academic Press).

Jallade, Sébastien (2011). "La Réinvention des Routes Incas: Représentations et Construction de la Mémoire au Pérou (2001-2011)", Droit et Cultures, Vol. 62, pp. 119-137.

Jowers, Kevin F. (2003). "International and National Legal Efforts to Protect Cultural Property: The 1970 Unesco Convention, the United States, and Mexico", The Texas International Law Journal, Vol. 38, pp. 145-171. 
Korstanje, M. Alejandra y García-Azcárate, Jorgelina (2007). "The Qhapaq Nan Project: A Critical View”, Archaeologies, Vol. 3 (No 2), pp. 116-131.

Krauss, Michael E. (2007). "Mass Language Extinction and Documentation: The Race Against Time”, en Miyaoka O., Sakiyama O. Y Krauss, Michael E. (edit.), The Vanishing Languages of The Pacific Rim (Oxford, Oxford University Press), pp. 3-23.

Labadi, Sophia (2013). Unesco, Cultural Heritage, and Outstanding Universal Value: Value-based Analyses of the World Heritage and Intangible Cultural Heritage Conventions (Lanham, Altamira Press).

Labadi, Sophia y Long, Colin (2010). Heritage and Globalization (New York, Routledge).

Langfield, Michele; Logan, William y Craith, Mairead Nic (2010). Cultural Diversity, Heritage and Human Rights: Intersections in Theory and Practice (New York, Routledge).

Losson, Pierre (2017). "The inscription of Qhapaq Nan on Unesco's World Heritage List: a comparative perspective from the daily press in six Latin American countries", en International Journal of Heritage Studies, Vol. 23, pp. 521-537.

Mead, Moko (1993). "The Maintenance of Heritage in a Fourth World Context: The Mãori Case", en Dark, Philip J.C. y Rose, Roger G. (edit.), Artistic Heritage in a Changing Pacific (Honolulu, University of Hawaii Press), pp. 223-231.

Merryman, J. H. (1986). "Two Ways of Thinking about Cultural Property", en The American Journal of International Law, Vol. 80 (No 4), pp. 831-853.

Meyer-Bisch, Patrice (2009). "On the 'Right to Heritage' - The innovative approach of articles 1 and 2 of the Faro Convention”, en Palmer, Robert, Heritage and Beyond (Strasbourg, Council of Europe), pp. 59-65.

Morales, Patrick (2013). "La Gentrificación en Cartagena, el Desalojo Neoliberal", en Revista Arcadia, febrero.

Nafziger, James A. R. (2008). "Cultural Heritage Law: The International Regime. Report of the Director of Studies of the English-Speaking Section of the Centre", en Nafziger, James A. R. y Scovazzi, Tullio (edit.) Le Patrimoine Culturel de l'Humanité $=$ The Cultural Heritage of Mankind (Leiden, Martinus Nijhoff Publishers), pp. 145-247. 
NAfZiger, James A. R. y Janis, Mark W. (2006). "The Development of International Cultural Law", en Proceedings of the Annual Meeting of the American Society of International Law, Vol. 100, pp. 317-323.

Nafziger, James; Paterson, Robert y Renteln, Alison (2010). Cultural Law: International, Comparative, and Indigenous (New York, Cambridge University Press).

OchoA Jiménez, María Julia (2012). "América Latina frente al Orden Jurídico Internacional sobre Cultura", en Revista Iberoamericana de Autogestión y Acción Comunal (No 60), pp. 125-145.

O'Keefe, Patrick (2007). Commentary on the 1970 Unesco Convention on the Means of Prohibiting and Preventing the Illicit Import, Export and Transfer of Ownership of Cultural Property (Leicester, Institute of Art and Law).

Oxford English Dictionary (2018). (Oxford, Oxford University Press).

Pérez-Prat, Luis (2014). "Observaciones sobre el Derecho al Patrimonio Cultural como Derecho Humano”, Periférica Internacional, Revista para el Análisis de la Cultura y el Territorio (No 15), pp. 319-342.

Prott, Lyndel V. (1989). "Problems of Private International Law for the Protection of the Cultural Heritage", Recueil des Cours de l'Académie de La Haye, Vol. 217, pp. 219-317.

Prott, Lyndel V. (2012). Strengths and Weaknesses of the 1970 Convention: An Evaluation 40 years after its Adoption (París, Unesco).

Prott, Lyndel V. y O’Keefe, Patrick J. (1992) “Cultural Heritage’ or 'Cultural Property'?”, International Journal of Cultural Property, Vol. 1 (No 2), pp. 307-320.

Real Academia Española (2014). Diccionario de la lengua española (edición en línea).

Roy, Olivier y Annicchino, Pasquale (2013). "Human Rights between Religions, Cultures, and Universality”, en Vrdoljak, Ana Filipa (edit.), The Cultural Dimension of Human Rights (Oxford, Oxford University Press), pp. 13-25.

Sánchez de Guzmán, Esther (2006). Entre el Juez Salomón y el Dios Sira: Decisiones Interculturales e Interés Superior del Niño (Bogotá, Gente Nueva).

SHAHEED, Farida (2011). Report of the Independent Expert in the Field of Cultural Rights (United Nations, A/HRC/17/38).

SHAHEED, Farida (2014). Memorialization Processes Report of the Special Rapporteur in the Field of Cultural Rights (United Nations, A/HRC/2 5/49). 
Stamatopoulou, Elsa (2007). Cultural Rights in International Law. Article 27 of the Universal Declaration of Human Rights and Beyond (Leiden, Martinus Nijhoff Publishers).

Steiner, Lasse y FreY, Bruno S. (2012). "Correcting the Imbalance of the World Heritage List: Did the Unesco Strategy Work?”, en Journal of International Organizations Studies, Vol. 3 (No 1), pp. 25-40.

The Economist (2012). "Booming Mongolia: Mine, all mine" (London, online ed., 21 January 2012).

Unesco (2001). Declaración Universal de la Unesco sobre la Diversidad Cultural. UnESCo (2008). Directrices prácticas para la aplicación de la Convención del Patrimonio Mundial WHC. 08/01.

UNESCO (2013). Inscription of Mongolian Calligraphy on the List of Intangible Cultural Heritage in Need of Urgent Safeguarding ITH/13/8.COM/7.A.9.

UNESCO (2014). Operational Directives for the Implementation of the Convention for the Safeguarding of the Intangible Cultural Heritage 5.GA.

UnESCO (2014b). Report of the Decisions adopted by the World Heritage WHC14/38.COM/16.

Unesco e Iccrom (2004). Monitoring World Heritage (París, Unesco).

United Nations (1960). Declaración sobre la Concesión de la Independencia a los Paises y Pueblos Coloniales GA Res 1514, XV.

United Nations (2007). Declaration on the Rights of Indigenous Peoples GA Res 61/295, A/RES/61/295.

Valderrama, Fernando (1995). A History of Unesco (París, Unesco).

\section{NORMAS}

Code du Patrimoine, version consolidée au 1 janvier 2018.

Convenio 169 de la OIT sobre Pueblos Indígenas y Tribales en Países Independientes (1989).

Convenio de Unidroit sobre los Bienes Culturales Robados o Exportados Ilícitamente (1995).

Convención para la Protección de los Bienes Culturales en caso de Conflicto Armado y Reglamento para la aplicación de la Convención (1954).

Convención para la Salvaguardia del Patrimonio Cultural Inmaterial (2003).

Convención sobre la Protección del Patrimonio Mundial Cultural y Natural (1972). 
Convención sobre las Medidas que Deben Adoptarse para Prohibir e Impedir la Importación, la Exportación y la Transferencia de Propiedad Ilícitas de Bienes Culturales (1970).

Convención sobre los Derechos del Niño (1989).

Declaración Universal de los Derechos Humanos (1948).

Framework Convention on the Value of Cultural Heritage for Society (2005).

Instrumento para la Protección de los Derechos de las Minorías de la Iniciativa de Europa Central (1994).

Pacto Internacional de Derechos Civiles y Políticos (1966).

Pacto Internacional de Derechos Económicos, Sociales y Culturales (1966).

Protocolo Adicional a la Convención Americana sobre Derechos Humanos en Materia de Derechos Económicos, Sociales y Culturales (1988).

\section{JURISPRUDENCIA}

African Commission on Human and Peoples' Rights con Republic of Kenya (2017). Corte Africana de Derechos Humanos y de los Pueblos, 26 de mayo de 2017. Comunidad Mayagna (Sumo) Awas Tingni con Nicaragua (2001). Corte Interamericana de Derechos Humanos, 31 de agosto de 2001.

Luis Antidio Anama Ramírez con Cabildo Indígena Yanacona, Resguardo Caquiona de Almaguer-Cauca- (2001). Corte Constitucional de Colombia, T-1022 de 20 de septiembre de 2001.

Álvaro de Jesús Torres Forero con Autoridades Tradicionales de la Comunidad Indigena Arhuaca de la Zona Oriental de la Sierra Nevada de Santa Marta (1998). Corte Constitucional de Colombia, SU-510 de 18 de septiembre de 1998. 